\title{
Masculinity, Moral Atmosphere, and Moral Functioning of High School Football Players
}

\author{
Jesse A. Steinfeldt, ${ }^{1}$ Leslie A. Rutkowski, ${ }^{1}$ Ellen L. Vaughan, ${ }^{1}$ \\ and Matthew C. Steinfeldt ${ }^{2}$ \\ ${ }^{1}$ Indiana University-Bloomington and ${ }^{2}$ Fort Lewis College
}

\begin{abstract}
In order to identify factors associated with on-field moral functioning among student athletes within the unique context of football, we examined masculine gender role conflict, moral atmosphere, and athletic identity. Using structural equation modeling to assess survey data from 204 high school football players, results demonstrated that moral atmosphere (i.e., the influence of coaches and teammates) was significantly associated with participants' process of on-field moral functioning across the levels of judgment, intention, and behavior. Neither masculine gender role conflict nor athletic identity significantly predicted moral functioning, but the results indicated that participants' identification with the athlete role significantly predicted conflict with socialized gender roles. Results suggest that in the aggressive and violent sport of football, coaches can have a direct influence on players' moral functioning process. Coaches can also have an indirect effect by influencing all the players so that a culture of ethical play can be cultivated among teammates and spread from the top down.
\end{abstract}

Keywords: masculine gender role conflict, student athletes, bracketed morality, athletic identity, masculinity socialization

"Sport, as a social practice, is actively involved in the individuals' socialization and one often highlights the role it plays in developing moral values such as support, fair play, solidarity, or cooperation" (Coulomb-Cabagno \& Rascle, 2006, p. 1981). This quote highlights the influential ability of sport to provide its participants with opportunities to experience personal growth and development. However, antisocial behaviors on the field (e.g., poor sportspersonship, unethical play, cheating) are aspects of competitive sport that undermines sport's ability to instill desirable values in young student athletes. Within the framework of bracketed morality theory (Bredemeier \& Shields, 1986), much attention has been paid to aspects of moral atmosphere in sport in an attempt to better understand what contributes to moral functioning on the fields of play. In addition to the role of moral

Jesse A. Steinfeldt, Leslie A. Rutkowski, and Ellen L. Vaughan are with the Department of Counseling and Educational Psychology, Indiana University, Bloomington, IN. Matthew C. Steinfeldt is with the Department of Athletics, Fort Lewis College, Durango, CO. 
atmosphere, studies have examined the effects of variables such as social influence or approval (Shields, LaVoi, Bredemeier, \& Power, 2007), professionalism (Visek \&Watson, 2005), goal orientation (Sage, Kavussanu, \& Duda, 2006; Stephens \& Kavanagh, 2003), motivational climate (Kavussanu, Roberts, \& Ntoumanis, 2002; Miller, Roberts, \& Ommundsen, 2005), team leadership and cohesion (Shields, Bredemeier, Gardner, \& Bostrom, 1995), and sex differences (Coulomb-Cabagno \& Rascle, 2006; Kavussanu, Stamp, Slade, \& Ring, 2009).

In spite of this emerging body of research, no studies to date have included gender role socialization as a potential contributor to this process of moral functioning in sport. Masculine gender role conflict, a negative psychological state induced by conflict stemming from socialized gender role expectations, has been empirically linked to a host of negative interpersonal and intrapersonal outcomes (O’Neil, 2008; O’Neil, Helms, Gable, David, \& Wrightsman, 1986). Recent research has investigated the relationship between gender role conflict and aggression toward others (Cohn, Jakupcak, Seibert, Hildebrandt, \& Zeichner, 2010; Cohn \& Zeichner, 2005), but this research has not yet been extended into the domain of sport. Because the sport of American football values, encourages, and sanctions instrumental aggressive behavior (Gage, 2008; Messner, 1990), this study intended to extend the findings of past researchers (e.g., Kavussanu et al., 2002) by examining masculine gender role conflict_-along with moral atmosphere and athletic identity - to identify factors associated with the moral functioning of high school student athletes within the unique context of football.

\section{Gender Role Conflict and Masculinity Socialization in Sport}

Similar to other psychosocial theories of masculinity socialization within the gender role strain paradigm (Pleck, 1995), gender role conflict theory emphasizes the social influences on masculine gender role expectations. Gender role conflict theory purports that attempts to conform to socialized gender role expectations produce psychological distress in men when they are unable to live up to these expectations (O’Neil, 2008). The conflict associated with the stress of gender role socialization produces interpersonal and intrapersonal strain that restricts men's ability to reach their full potential, and the resulting psychological state is referred to as gender role conflict (O’Neil et al., 1986). "Gender role conflict is hypothesized to occur cognitively, emotionally, behaviorally, and unconsciously and include personal experiences of gender role restrictions, devaluations, and violations" (O'Neil, 2008, p. 366). Four distinct patterns of this gender role conflict have been identified in research and through clinical work with men: (a) success, power, and competition (SPC), which is referred to as the need for success and achievement (NSA) in the adolescent version of the Gender Role Conflict Scale (GRCS-A; Blazina, Pisecco, \& O'Neil, 2005); (b) restrictive emotionality (RE); (c) restrictive affectionate behavior between men (RABBM), which is referred to as restricted affection between men (RAM) in the adolescent version; and (d) conflict between work and family relations (CBWFR), which is referred to as conflict between work, school, and family (CWSF) in the adolescent version. Each pattern of gender role conflict can have differential and independent effects on specific outcomes, across different contexts.

In their content analysis of a decade of publications in the journal Psychology of Men and Masculinity, Wong, Steinfeldt, Speight, and Hickman (2010) reported 
that gender role conflict theory was the most frequently used gender theoretical orientation employed by articles in this flagship journal of the Society for the Psychological Study of Men and Masculinity (SPSMM). To this end, the Gender Role Conflict Scale (GRCS; O'Neil et al., 1986) has been used in over 250 empirical studies since its creation (O'Neil, 2010). The associated body of research has consistently demonstrated the relationship between gender role conflict and a host of negative outcomes, including depression (Blazina \& Watkins, 1996; Shepard, 2002), anxiety (Cournoyer \& Mahalik, 1995; Wong, Pituch, \& Rochlen, 2006), substance abuse (Monk \& Ricciardelli, 2003), decreased marital satisfaction (Rochlen \& Mahalik, 2004), and a variety of additional negative intrapersonal and interpersonal outcomes.

In his comprehensive review of 25 years of gender role conflict research, O'Neil (2008) identified a need for research that explores this prominent theory of masculinity socialization across different contexts. The context of sport represents a fertile field for examining masculine gender role expectations that result from the unique gender socialization processes operating within this domain. Sports represent an influential domain in which boys can learn the social expectations, behaviors, and standards associated with being a man (Greendorfer, 1993). However, due to the structure of competitive sport, the masculinity expectations and standards learned in sport are often perceived as conditional. Young men who experience success in sport receive peer acceptance and attention from others (e.g., coaches, family, spectators) through their athletic accomplishments (Messner, 1992). These young men become aware that conditional acceptance is based on winning, which can contribute to the development of narrow definitions of success (i.e., being a winner). Subsequently, a young athlete's dominant self-image often focuses on the glorified athletic self, an identity contingent on winning and continuing to win (Messner, 1992).

Athletes who strongly identify with this athlete aspect of the self often develop a salient athletic identity (Brewer, Van Raalte, \& Linder, 1993). While identification with the athlete role is not inherently problematic, an overidentification with the athlete role can have deleterious consequences when it forecloses exploration of other aspects of one's identity (e.g., academic, social; Miller \& Kerr, 2002). An overly salient athletic identity has been linked to negative outcomes, including decreased academic performance and difficulties transitioning out of sport after retirement (Brewer \& Cornelius, 2001; Brewer et al., 1993; Grove, Lavallee, \& Gordon, 1997). In addition, recent interdisciplinary research of masculinity in sport has linked higher levels of athletic identity to negative attitudes toward help seeking and to higher levels of gender role conflict among football players (Steinfeldt \& Steinfeldt, 2010; Steinfeldt, Steinfeldt, England, \& Speight, 2009). Specifically, Steinfeldt et al. (2009) found that football players who reported higher levels of gender role conflict also reported higher levels of athletic identity and more negative attitudes toward seeking help. Similarly, in their examination of high school football players, Steinfeldt and Steinfeldt (2010) reported that athletic identity significantly predicted higher levels of gender role conflict. Thus, emerging research has demonstrated that athletic identity and gender role conflict are related constructs that can impact the psychosocial functioning of student athletes off the field (e.g., help seeking). However, no research to date has examined the impact of masculine gender role conflict on the field. 


\section{Contextual Determinants of Moral Functioning in Sport}

In their experimental study on gender role conflict and aggression toward others, Cohn et al. (2010) reported that the significant relationship between restrictive emotionality and aggression was mediated by higher levels of emotional dysregulation. However, because laboratory setting results such as these are often difficult to generalize, there is a need for research to examine if a relationship exists between gender role conflict and antisocial behaviors (e.g., behaving aggressively toward another person) in real-life settings. Sport represents an interesting context to examine this dynamic because a variety of antisocial behaviors exist in sport (e.g., Shields \& Bredemeier, 2007). Research that has investigated antisocial behaviors among athletes has frequently examined moral atmosphere as a contributing variable (Shields \& Bredemeier, 2007), and much of this research has operated from the theoretical framework of bracketed morality (Bredemeier \& Shields, 1986). The concept of bracketed morality suggests that sport facilitates a temporary suspension of everyday moral functioning. The rules, values, and structure of competitive sport serve to create an environment in which athletes engage in a process where their on-field behaviors may differ from the process of moral functioning they might otherwise use off the field. For example, contact sports reward and require instrumental aggression, despite the fact that this aggressive behavior runs counter to social norms that proscribe appropriate interpersonal interactions off the field (Stephens, 1998). Thus, the decision to physically knock down another player may be justified in a game, particularly when it provides a competitive advantage, but this behavior would not be appropriate in another setting (e.g., restaurant, classroom). Although rules in sport exist to maintain the integrity of the game, there are often gray areas of interpretation (e.g., what constitutes a legal hit in football, what actions might be considered strategic but not necessarily in the spirit of the rules) that allow for diverse moral functioning processes to exist on the field and potentially undermine the ethos of the game.

According to Shields and Bredemeier (2007), one model consistently used to empirically examine moral functioning in sport is Rest's $(1983,1984)$ model of moral action. This model operates under the premise that in each moral action, there are four processes: (a) interpreting the situation, (b) forming a moral judgment about the appropriate thing to do, (c) deciding what one intends to do by selecting among competing values, and (d) engaging in the behavior. These processes are interactive, and can be influenced by a variety of factors. The majority of the moral functioning research in sport has examined the second (i.e., judgment) and third (i.e., intention) processes of Rest's model (Shields \& Bredemeier, 2007), but research has also included the fourth process (i.e., behavior; e.g., Kavussanu et al., 2002). Kavussanu et al. (2002) conducted a study to determine the role of moral atmosphere and perceived motivational climate on the on-court moral functioning of 199 college basketball players. The authors used Rest's model $(1983,1984)$ to assess moral functioning across four on-court basketball-specific scenarios (i.e., intimidation, risk injury to opponent, cheating, intentionally injure opponent). Results indicated that perceived motivational climate did not significantly predict on-court moral functioning, but moral atmosphere was a significant predictor. Thus, the college basketball players in this study indicated that the influence of coaches and teammates impacted their moral judgment, moral intentions, and moral behaviors on the basketball court (Kavussanu et al., 2002). 
Similar to the Kavussanu et al. (2002) study, much of the research into moral atmosphere in athletics has investigated its role in moral functioning within sports such as soccer, handball, basketball, hockey, volleyball, and others. In an examination of 676 young athletes from six different sports, Shields et al. (2007) included a small sample of football players, and found that antisocial sports behaviors occurred more frequently among football players in comparison with athletes in medium-contact sports. Despite the prevalence of football in U.S. sports culture, there have not been any exclusive examinations of moral functioning in the contact sport of football, even though football's aggressive and violent nature (e.g., Gage, 2008; Messner, 1990) provides opportunities for ambiguity about what constitutes antisocial behavior on the field.

\section{Current Study}

The purpose of this study was to evaluate potential variables that contribute to moral functioning within the unique context of football. Based on past research in the fields of sport psychology and the scientific study of men and masculinity, we created an interdisciplinary conceptual model (see Figure 1), discussed subsequently. Our model used the design of Kavussanu et al. (2002), based on our desire to extend their study's framework into the relatively unexamined domain of football. We employed Rest's $(1983,1984)$ model of moral action, with Gender Role Conflict substituted for the Motivational Climate variable used in Kavussanu et al.'s (2002) study, and we added athletic identity as an additional predictor variable for the moral functioning construct. Our goal was to determine if tenets of this prominent theory of masculinity socialization (e.g., gender role conflict), in combination with moral atmosphere (i.e., influence of teammates and coaches) and athletic identity, would predict on-field moral functioning among high school football players. We discuss the hypothesized model specifically using the numbered directional paths in Figure 1. Our first hypothesis was that a weaker moral atmosphere (i.e., participant perceptions that teammates or coaches condone antisocial behavior on the field) would predict judgments, intentions, and behaviors that reflect lower levels of moral functioning on the football field (path 1). This hypothesis was based on consistent findings in the literature that demonstrate that lower levels of moral atmosphere contribute to lower levels of moral functioning in other sports (Kavussanu et al., 2002; Kavussanu et al., 2009; Miller et al., 2005; Shields \& Bredemeier, 2007; Shields et al., 2007; Stephens \& Kavanagh, 2003).

According to Gage (2008), "research has found that reinforcement of masculine gender norms through strict football rituals in team practices creates a behavioral repertoire that may be extended to other contexts" (p. 1016). Thus, our second hypothesis was that higher levels of gender role conflict would predict a greater likelihood that a player would demonstrate decreased moral functioning on the field (Path 3), and our third hypothesis was that gender role conflict would mediate the relationship between moral atmosphere and moral functioning (Paths 2 and 3). Research on gender role conflict has consistently shown that men who restrict their emotional repertoire are more likely to experience negative outcomes, including aggression toward others (Cohn et al., 2010), lack of male friendship and intimacy, and a variety of other interpersonal difficulties (see O'Neil, 2008). Furthermore, men who place an inordinate emphasis on success and achievement through 


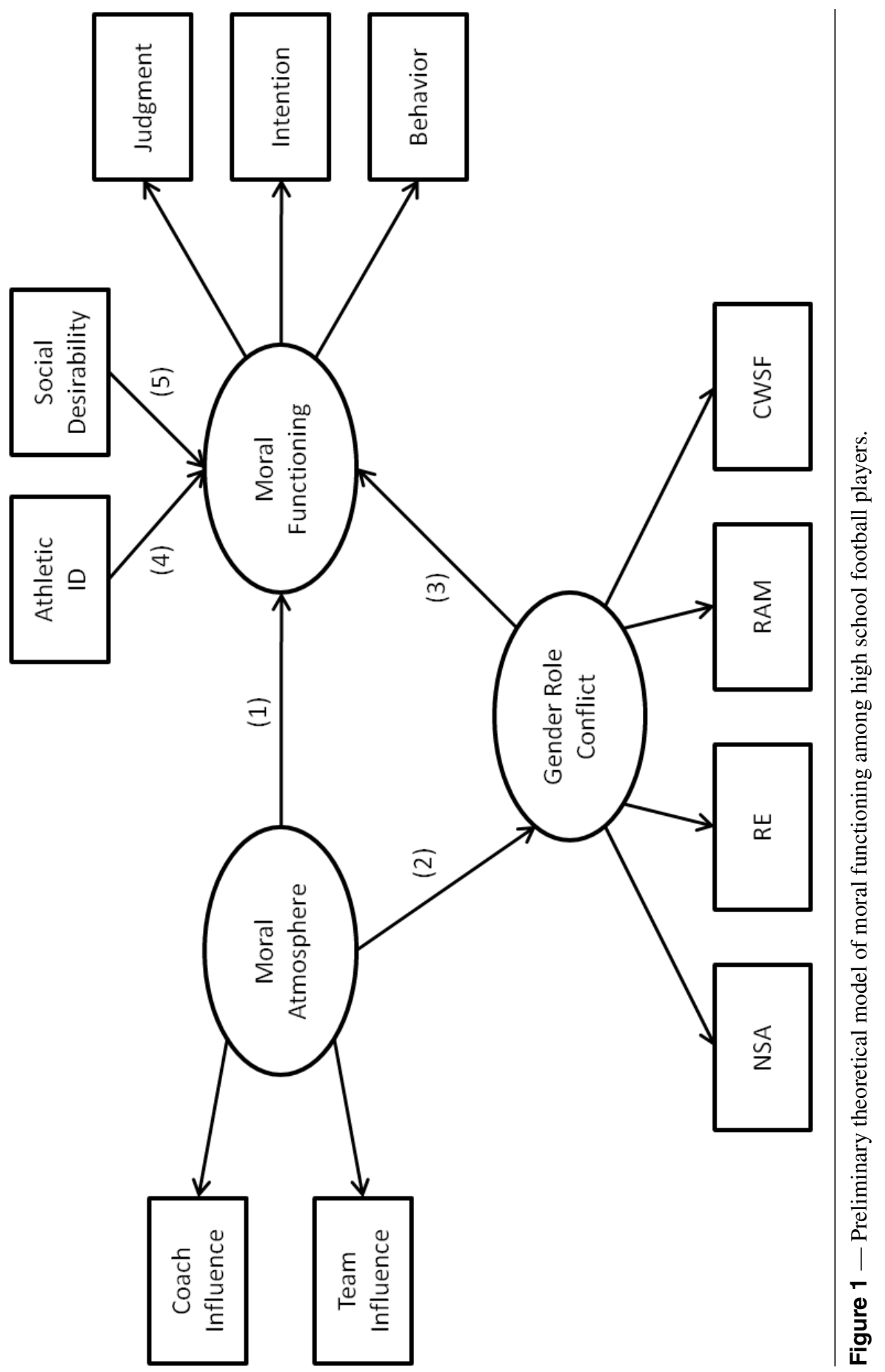


competitive, rather than cooperative, means often experience negative intrapersonal and interpersonal outcomes (O'Neil, 2008). Accordingly, we predicted that higher levels of gender role conflict - as exemplified by the aforementioned aspects of this constraining psychological construct-would contribute to a young man's decision to sacrifice the ethos of the game and engage in judgments, intentions, and behaviors that condone antisocial behaviors on the field.

Finally, in addition to controlling for social desirability in our model (Path 5 ), our fourth hypothesis was that higher levels of athletic identity would predict lower levels of moral functioning (i.e., moral judgment, intentions, and behaviors that support antisocial behaviors on the field of play; Path 4). This hypothesis was based on the premise that a football player who overidentifies with the athlete aspect of his self-identity might more readily compromise the integrity of the game to maintain that conditional and glorified athletic self that results from the emphasis on winning that sport fosters (Messner, 1992). In sum, based on our four hypotheses, our model predicted that the weaker a player perceives the moral atmosphere on his team to be, the more likely he will be to demonstrate lower moral functioning on the field across the dimensions of judgment, intention, and behavior.

\section{Method}

\section{Participants}

The participants in this study were 204 high school football players between 14 and 18 years of age who attended one of three high schools in a city in the U.S. Midwest. The average age of the participants was $15.73(S D=1.26)$, and the participants consisted of 47 freshmen, 59 sophomores, 41 juniors, and 57 seniors. The sample self-identified their race as White (83\%), Black (3\%), Multiracial (3\%), Asian American (3\%), Hispanic (3\%), and American Indian (3\%). Two percent of the sample did not report a racial identification, and the racial composition of the sample was consistent with the racial demographics of the community. Finally, the sample had an average overall GPA of $3.19(S D=0.60)$.

\section{Procedures}

Research was conducted in compliance with institutional review board approval from the first author's host institution. In addition, the school district required its own review process for access to the participating high schools. School system administrators, school principals, athletic directors, and coaches were contacted, and they subsequently agreed to make players available to participate in the study. The survey packet was distributed to participants during a team meeting, attended by the first author, where parental consent forms were distributed to players and were taken home. At a later team meeting, participants who returned with signed parental consent and assent forms were provided the opportunity for voluntary participation. After obtaining informed consent and assent forms from parents and participants, the first author distributed the questionnaires and an information sheet. Participants were assured of anonymity and were informed that all their data would be kept confidential and in a safe locked location. Participants took approximately 15-20 min to complete the survey packet. To ensure voluntary participation, 
participants were informed that if they did not want to participate in the study, they could write in their playbooks and turn in a blank survey packet at the end. Two players turned in completed parental consent forms but opted not to participate and return a completed survey, giving a response rate of $99 \%$.

\section{Measures}

Gender Role Conflict. The Gender Role Conflict Scale-Adolescent version (GRSC-A; Blazina et al., 2005) is a 29-item self-report instrument that uses a sixpoint Likert-type scale with possible responses ranging from 1 (strongly disagree) to 6 (strongly agree). The GRCS-A, adapted from the adult version of the GRCS (O'Neil et al., 1986), is designed to assess dimensions of masculine role conflict among adolescents. The adolescent version modified aspects of the original GRCS to make it appropriate for use with adolescents. For example, items on the GRCS-A were rephrased to shift the focus from sexual to personal relationships, and school activities were added as a source of conflict. These adaptations were made in an effort to maintain the integrity of the original scale, yet make the items relevant to adolescent males (see Blazina et al., 2005). The gender role conflict measured by the GRCS-A occurs when rigid or restrictive gender roles, which are learned through a socialization process, result in a restriction, devaluation, or violation of self or others (O'Neil et al., 1986). Similar to the adult version of the GRCS, the GRCS-A has four subscales:

1. Need for Success and Achievement (NSA; e.g., "Doing well all the time is important to me" and "I strive to be more successful than others"), measured by six items.

2. Restricted Emotionality (RE; e.g., "I have difficulty expressing my emotional needs to others" and "I often have trouble finding words that describe how I am feeling"), measured by nine items.

3. Restricted Affection Between Men (RAM; e.g., "Affection with other men makes me tense" and "Being very personal with other men makes me feel anxious"), measured by seven items.

4. Conflict Between Work, School, and Family (CWSF; e.g., "My work or school often disrupts other parts of my life" and "Overwork and stress, caused by a need to achieve on the job or in school, affects or hurts my life"), measured by seven items.

These four subscales represent the four patterns of conflict with masculine gender role expectations identified among adolescent males, and higher scores represent higher levels of gender role conflict.

Validity for the GRCS-A has been established by its significant relationship to another psychometrically validated adolescent masculinity scale, the Male Role Attitude Scale (Thompson \& Pleck, 1986). In addition, Blazina et al. (2005) reported convergent validity based on the GRCS-A and the original GRCS factors being positively correlated with the theoretically corresponding scales. In support of the scale's reliability, Steinfeldt and Steinfeldt (2010) examined gender role conflict among high school football players, and reported the following internal consistency coefficients, as measured by Cronbach's coefficient alpha: NSA = .72; RE = .77; 
RAM $=.76$; and CWFS $=.73$. Given the growing obsolescence of coefficient alpha as a measure of internal consistency, we adopted a more contemporary approach for the current study and used Guttman's (1945) $\lambda_{2}$, which is known to be a better lower bound to reliability, since $\alpha \leq \lambda_{2} \leq \rho_{X, X}$ (Sijtsma, 2009) and may be a better bound than the greatest lower bound, $\rho_{\mathrm{glb}}$, when sample sizes are relatively small (Ten Berge \& Sočan, 2004). To compare with the previously reported internal consistency estimates, we also report coefficient alpha. The internal consistency coefficients for the current study were as follows: NSA: $\alpha=.59, \lambda_{2}=.60$; RE: $\alpha=$ $.83, \lambda_{2}=.83$; RAM: $\alpha=.77, \lambda_{2}=.77$; and CWFS: $\alpha=.74, \lambda_{2}=.74$.

Athletic Identity. The Athletic Identity Measurement Scale (AIMS; Brewer \& Cornelius, 2001) is a seven-item, self-report instrument that uses a seven-point Likert-type scale to measure the strength and exclusivity of identification with the athlete role. Possible responses range from 1 (strongly disagree) to 7 (strongly agree). Items (e.g., "Sport is the most important part of my life" or "I consider myself an athlete") rate the extent to which respondents agree with statements about cognitive, affective, and behavioral aspects of identification with the athlete role. Higher AIMS scores indicate a stronger and more exclusive identification with the athlete role. Steinfeldt and Steinfeldt (2010) reported an internal consistency of .81 with high school football players, and the coefficient alpha was .78 for the AIMS in the current study. Similarly, $\lambda_{2}=.80$ in the current sample. Differences in athletic identity across levels of athletic participation provide support for the construct validity of AIMS. Compared with the scores of student athletes, nonathletes reported significantly lower AIMS scores (Brewer et al., 1993; Cornelius, 1995).

Moral Functioning. Moral functioning was assessed by adapting a measure used by Kavussanu et al. (2002) to assess moral functioning in their study of college basketball players.

Through extensive consultation with academics, sport psychologists, and high school and college football coaches, the four scenarios in this measure were revised to be football specific. The adapted scenarios used in this study were as follows.

1. Intimidate: During a critical football game, you have just knocked your opponent to the ground (after making a tackle or a block). To intimidate him when the referees are not looking, you push off of him to get yourself up off the ground.

2. Risk Injuring Opponent: In the last minute of a critical football game, your team is leading by four points. However, the opposing team is driving toward your end zone. You get past the offensive lineman as you rush the quarterback, but you begin stumbling to the ground. The only way to get to the quarterback is to dive at his knees from the blind side. You have to decide whether to risk injuring the player to get the sack that potentially clinches the victory for your team.

3. Cheating: It is the last minute of a critical football game, and your team is leading by two points. The other team has just completed its third pass in a row in their no huddle offense. They have crossed midfield and have momentum. To give your team rest and to make defensive substitutions, you stay on the ground after a play and fake an injury to stop the clock. 
4. Intentionally Injure Opponent: You are in a critical football game. After an interception (or during a punt/kick return), you are trailing far behind the play. You are running alongside the other team's best player, who is playing with bruised ribs. Because the player is merely jogging down the field, you can take him out of the game by hitting him with a ferocious blindside shot without being caught by the referee.

These scenarios were assessed across three levels (i.e., judgment, intention, behavior) of Rest's $(1983,1984)$ model. Similar to past researchers (e.g., Shields \& Bredemeier, 2007), we did not assess the first level (i.e., interpretation) of Rest's model. For the second level, judgment, participants were asked after each scenario to judge how appropriate they deemed the behavior to be. The items used a five-point Likert-type scale with possible responses ranging from 1 (never appropriate) to 5 (always appropriate). The internal consistency coefficient for the Moral Judgment scale was $\alpha=.73, \lambda_{2}=.74$, consistent with the coefficient alpha of .67 for this scale reported by Kavussanu et al. (2002). A similar procedure was followed for the intention and behavior levels of Rest's model. Participants were asked if they would engage in the behavior (i.e., intention), and were then asked how often they had actually engaged in the behavior in the past five games (i.e., behavior). Similar to the moral judgment dimension, both moral intention and moral behavior were assessed using the four scenarios and a five-point Likert-type scale with possible responses ranging from 1 (never) to 5 (very often). The internal consistency coefficients for the Moral Intention scale $\left(\mathrm{g}=.71, \lambda_{2}=.72\right)$ and the Moral Behavior scale $\left(\alpha=.76, \lambda_{2}=.77\right)$ were also consistent with the coefficients alpha of .72 and .70 reported by Kavussanu et al. (2002) on the respective dimensions.

Moral Atmosphere. Moral atmosphere can be conceptualized as the collective norms that contribute to the unique construction of a group's moral climate (Shields et al., 2007). Consistent with past research, moral atmosphere in this study was comprised of two dimensions: coach influence and teammate influence. Across the four scenarios of moral functioning, participants were asked the degree to which they thought their coach would approve of the behavior in the scenario, if it was necessary to win. A five-point Likert-type scale was used, with possible responses ranging from 1 (strongly discourage) to 5 (strongly encourage). To assess teammate influence across the four scenarios, participants were asked how many of their teammates they thought would engage in the described behavior. A fivepoint Likert-type scale was used, with possible responses including 1 (none of the players), 2 (a few players), 3 (about half of the players), 4 (most of the players), and 5 (everyone on the team). The internal consistency coefficients were $\alpha=.70$, $\lambda_{2}=.71$, for coach influence and $\alpha=.68, \lambda_{2}=.70$, for teammate influence, and were thus reasonably consistent with the coefficients alpha of .82 and .70 reported by Kavussanu et al. (2002) on the respective scales.

For their model that assessed the moral functioning and moral atmosphere variables, Kavussanu et al. (2002) used a multitrait-multimethod (MTMM) analysis to validate the two indicators (i.e., coach, teammate) of moral atmosphere and the three indicators (i.e., judgment, intention, behavior) of moral functioning across the four scenarios. Utilizing confirmatory factor analysis and structural equation modeling, the results of their MTMM analysis demonstrated support for this model. Thus, results suggest that this model is appropriate to use as a valid measure for 
assessing moral atmosphere and moral functioning in sport, and the results of this current study's analyses indicate that the adaptations we made resulted in a model that statistically significantly fit the data.

Social Desirability. Because people may attempt to portray themselves in a favorable light when responding to questions about moral variables, it is necessary to include a measure of social desirability in an effort to attempt to control for this potential bias (Sage et al., 2006). Thus, a short version of the Marlowe-Crowne Social Desirability Scale (Strahan \& Gerbasi, 1972) was included to determine if student athletes responded to questions in a socially desirable manner. The scale contains 10 items that describe socially desirable attributes (e.g., "I never resent being asked to return a favor"), and respondents are asked to indicate if the statement is true or false, as it relates to them personally. If a participant responded in a socially desirable manner, they received a score of 1 on that item (and a score of 0 was awarded otherwise).

\section{Item Parceling}

Based on our analysis strategy (i.e., structural equation modeling) discussed subsequently, an ideal model would incorporate individual indicators of each dimension and construct. For example, the athletic identity construct would be indicated by the seven questionnaire items that tap that latent construct. Unfortunately, our sample size precluded this approach, which would have resulted in as many as 13 latent variables, including second-order factor analyses for gender role conflict, moral atmosphere, and moral functioning. Instead, we chose an item-parceling scheme that used adequately fitting confirmatory factor analyses to justify combining items into single indicators. Specifically, nonsignificant chi-squares and acceptably small normalized residuals $(<2.00)$ were used as criteria for judging acceptable fit of the scale. Given that our interest was principally on the relations among latent variables and not necessarily on the relations among items, we found a parceling approach reasonably justified (Little, Cunningham, Shahar, \& Widaman, 2002).

Based on suitable confirmatory factor analysis results, the following measures were comprised of their associated items: NSA, RE, RAM, CWSF, athletic identity, coach influence, teammate influence, social desirability, moral judgment, moral intention, and moral behavior. All item parcels used sums across the relevant items to create scores for the resultant indicators. Only moral judgment did not include all of its component items. In particular, poor functioning of the moral judgment item in Scenario 2 (injuring a player) resulted in its removal from the scale. This left three items in the moral judgment scale: judgments about intimidating another player, cheating, and intentionally injuring another player.

\section{Analysis Strategy}

To test the hypothesized model of relationships between gender role conflict, moral atmosphere, athletic identity, and moral functioning, we used a structural equation modeling strategy with a maximum likelihood estimator and bootstrapped standard errors. To establish identification of the hypothesized model, we employed the recommended methods of McDonald and Ho (2002). To test the hypothesis that gender role conflict mediates the relationship between moral atmosphere and moral 
functioning, we use a method, described in MacKinnon, Lockwood, and Williams (2004) and implemented in MPlus (Muthén \& Muthén, 2010). In particular, the standard errors for the product of the path coefficients of interest were generated using the bias corrected bootstrap method with 1000 bootstrap draws. Model fit was evaluated based on the chi-square test (nonsignificant), the root mean squared error of approximation (RMSEA) $(<0.05)$ with standard errors, and an examination of normalized residuals $(<2.0)$. Further, we examined the possibility of model misspecification via the expected parameter change in combination with the modification index (MI) and the power of the MI test (Saris, Satorra, \& van der Veld, 2009). We set a minimum modification index value of 1.00 and a model misspecification value $(\delta)$ of .10. All models were fit with Mplus 6.0 (Muthén \& Muthén, 2010).

\section{Results}

\section{Influence on Process of Moral Functioning}

Using traditional measures of fit, the original theoretical model (see Figure 1) fit the data poorly $\left(\chi^{2}{ }_{31}=62.19, p<.001, \mathrm{CFI}=0.96, \mathrm{TLI}=0.94, \mathrm{RMSEA}=0.070\right.$, $95 \%$ CI [0.044, 0.095]). Based on an inspection of the residuals and previous research that documented different functioning of the NSA construct and scale (e.g., Steinfeldt \& Steinfeldt, 2010; Watts \& Borders, 2005), we eliminated the NSA measure from subsequent models. The modified model that eliminated NSA fit the data well according to traditional measures of model fit $\left(\chi^{2}{ }_{29}=41.56, p=\right.$ $.06, \mathrm{CFI}=0.98, \mathrm{TLI}=0.98, \mathrm{RMSEA}=0.046,95 \% \mathrm{CI}[0.000,0.076]$, all residuals $<2.0$ ). An investigation of model misspecification (Saris et al., 2009) indicated two theoretically tenable misspecifications including the regression of Gender Role Conflict on Athletic Identity (95\% $\left.\mathrm{CI}_{\mathrm{EPC}}[0.03,0.20], 1-\beta=.38\right)$ and correlated errors between Coach Influence and Teammate Influence $\left(95 \% \mathrm{CI}_{\mathrm{EPC}}[21.95\right.$, $125.58], 1-\beta=.95)$. Both modifications were considered; however, the model that included correlated errors between Coach Influence and Teammate Influence would not converge and was therefore rejected as a possibility. The model that included the regression of GRC on Athletic Identity fit the data well $\left(\chi_{28}^{2}=34.89, p=.17\right.$, $\mathrm{CFI}=0.99, \mathrm{TLI}=0.99, \mathrm{RMSEA}=0.035,95 \% \mathrm{CI}[0.000,0.068]$, all residuals $<$ 2.0) and is the model that we reported.

Figure 2 presents standardized regression weights and the significance for each path of the final model. Given our interest in the relationships among the latent variables and the exogenous observed variables, we do not discuss the measurement model results. The paths from Social Desirability and Athletic Identity to Moral Functioning were not significant. None of the latent paths to or from Gender Role Conflict were significant. Further, the indirect effect of Moral Atmosphere on Moral Functioning, as mediated by Gender Role Conflict was also not significant ( $\beta_{\text {indirect }}$ $=0.00, p=.99$ ). These findings suggest that experiencing conflict with socialized masculine role expectations was not significantly related to moral functioning on the field of play, nor was it related to the perceived moral atmosphere. As hypothesized, the model demonstrated significant paths between Moral Atmosphere and Moral Functioning $(\beta=0.93, p<.001)$. Thus, high school football players who reported that their teammates and their coach had engaged in or would encourage antisocial behaviors on the field were more likely to have judged these acts as appropriate, 


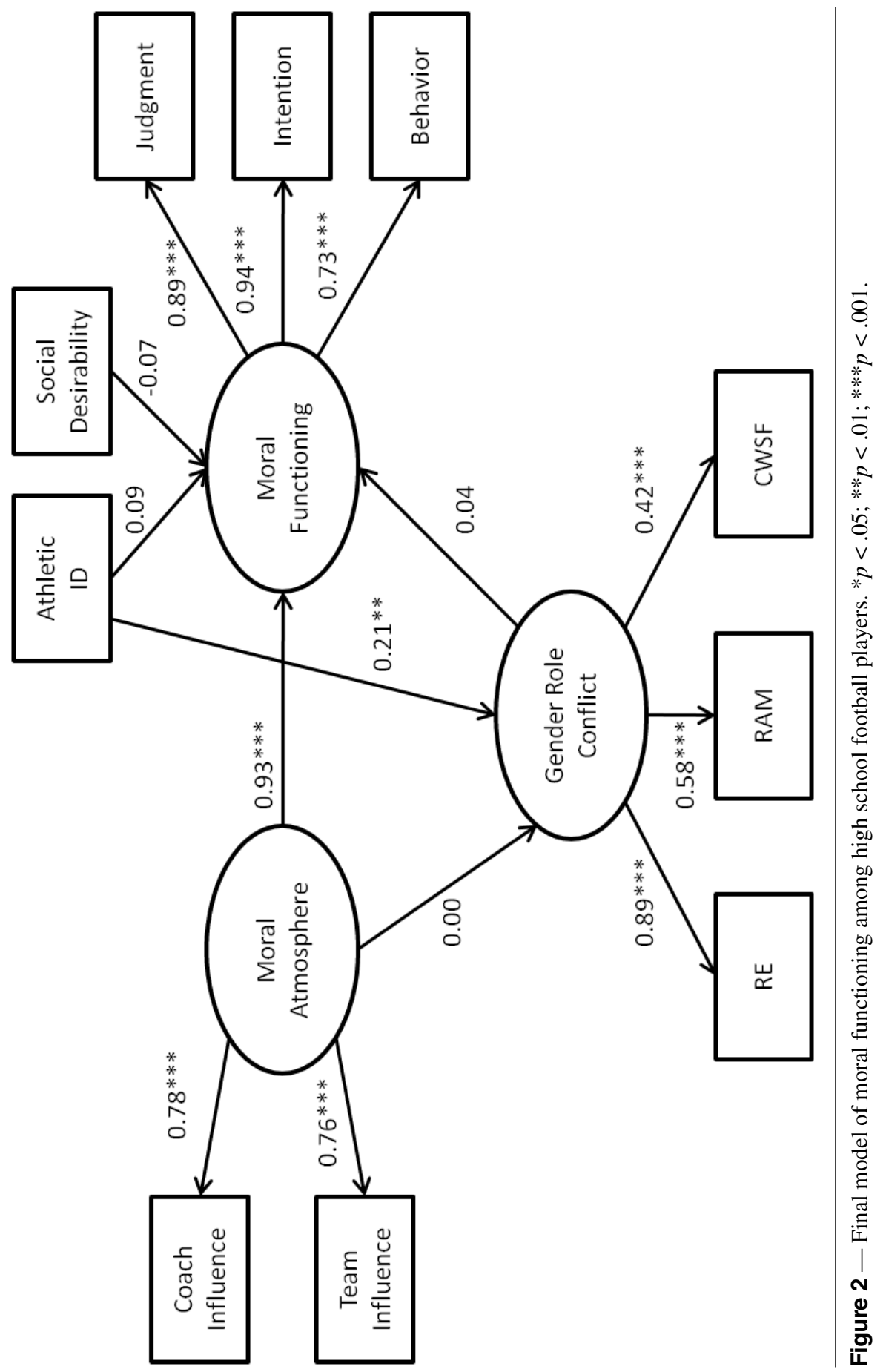


disclosed their intention to act in this manner, and engaged in these behaviors. Of particular interest was a significant finding of a positive effect for Athletic Identity on Gender Role Conflict $(\beta=0.21, p=.02)$. We expand on the implications of this finding in the discussion. Overall, these results indicate that a moral atmosphere that condones on-field antisocial behaviors is strongly and positively related to moral functioning that reflects judgments, intentions, and behaviors that support antisocial behaviors among adolescent football players on the field of play.

\section{Discussion}

\section{Role of Moral Atmosphere}

The results of this study indicated that that moral atmosphere (but not gender role conflict nor athletic identity) significantly predicted the on-field moral functioning of high school football players across three levels (i.e., judgment, intention, behavior) of Rest's $(1983,1984)$ model, and across the on-field scenarios of intimidation, risking injury to an opponent, cheating, and intentionally injuring an opponent. Despite these results, it is noteworthy to mention that, similar to the findings of Shields et al. (2007), the self-reported frequency of football players engaging in these antisocial behaviors was relatively low (see Table 1). However, the finding that coaches and teammates wielded great influence over players' process of moral functioning is consistent with the findings of Kavussanu et al. (2002), along with much of the literature on moral functioning in sport (see Shields \& Bredemeier, 2007). Thus, this study was reasonably successful in its attempt to extend the framework of Kavussanu et al. (2002) into the unique context of football.

The finding that moral atmosphere significantly predicted moral functioning has practical implications due to the fact that sport places decision-making power and moral responsibility largely into the hands of coaches (Bredemeier \& Shields, 1986). Similarly, Kavussanu et al. (2002) concluded that the results of their study "highlight the importance of the athlete's own team environment on his or her moral functioning and suggest that if we want to eliminate inappropriate action in sport, we need to start intervening at the level of the coach" (p. 363). Football is a contact sport that requires players to be relatively violent and aggressive to be

\section{Table 1 Responses and Frequencies of On-Field Antisocial Behaviors of High School Football Players $(N=204)$}

\begin{tabular}{lccccc}
\hline Behavior & Never & Rarely & Sometimes & Often & Always \\
\hline Intimidate opponent & 115 & 34 & 27 & 20 & 8 \\
& $56 \%$ & $17 \%$ & $13 \%$ & $10 \%$ & $4 \%$ \\
\cline { 2 - 6 } Risk injuring opponent & 98 & 45 & 32 & 18 & 11 \\
& $48 \%$ & $22 \%$ & $16 \%$ & $9 \%$ & $5 \%$ \\
\cline { 2 - 6 } Cheat & 170 & 21 & 7 & 1 & 5 \\
& $83 \%$ & $10 \%$ & $3 \%$ & $1 \%$ & $3 \%$ \\
\hline \multirow{3}{*}{ Intentionally injure opponent } & 139 & 30 & 13 & 11 & 11 \\
& $68 \%$ & $15 \%$ & $6 \%$ & $5 \%$ & $5 \%$ \\
\hline
\end{tabular}


successful (Gage, 2008; Messner, 1990). Thus, it is important that football coaches fully understand the impact they have on cultivating an environment wherein players understand how to aggress appropriately on the field (e.g., play within the rules and the ethos of the game), and that they should engage in these behaviors only on the field. That is, coaches expect their players to be violent and aggressive on the field (e.g., tackling someone, driving their opponent off the line of scrimmage), yet they also expect their players to "flip the switch" and find ways to be accountable and responsible young men off the field (Steinfeldt et al., in press). Subsequently, the results of this study can help coaches understand the importance of helping players learn to compartmentalize these behaviors (i.e., only engage in these behaviors on the field, and do not transfer these behaviors to off-field situations). A football player who does not understand how to compartmentalize these behaviors may be likely to engage in antisocial behaviors (e.g., bullying, fighting) off the field as well (Kreager, 2007). Future research should continue to explore how moral atmosphere and other variables influence the process of compartmentalizing aggressive behaviors, particularly in contact sports.

\section{Role of Gender Role Conflict and Athletic Identity}

Despite our second and third hypotheses to the contrary, gender role conflict did not significantly predict moral functioning, nor did it mediate the significant relationship between moral atmosphere and moral functioning found in this study. Gender role conflict has been consistently and robustly linked to a host of negative off-field outcomes (e.g., O'Neil, 2008; O’Neil et al., 1986), but this construct did not predict any of the on-field outcomes that we hypothesized. One interesting contributor to the Gender Role Conflict Scale's (O’Neil et al., 1986) nonsignificant predictive ability in this study was the Need for Success and Achievement (NSA) subscale. Specifically, poor CFA model fit led to our decision to drop the NSA subscale from the final model. Providing theoretical support for this decision, previous research (e.g., Steinfeldt \& Steinfeldt, 2010; Watts \& Borders, 2005) has demonstrated that the GRCS-A's (Blazina et al., 2005) NSA subscale has not operated similarly to the Success, Power, and Competition (SPC) subscale of the adult version of the GRCS. This previous research suggests that because NSA may operate developmentally as a specific (rather than global) construct among adolescents, NSA may not inherently contribute to negative outcomes in the same way SPC has been demonstrated to do in adults (e.g., O'Neil, 2008). Thus, while nonsignficant results (or results that do not confirm tenets of a given theory) are not always celebrated or even reported, such findings can provide the impetus for reflection and further contextual investigation into theoretical constructs. Researchers, particularly those in the scientific study of men and masculinity, can use the results of this study to engage in further research into the developmental nature of an adolescent's experience with socialized masculine roles.

In spite of these nonsignificant findings, future research would benefit from examining the nature of masculinity socialization within the unique context of sport to determine how socialized sport-specific masculinity messages (e.g., be tough, be aggressive, dominate your opponent, win-at-all-costs; e.g., Messner, 1992, 2002; Whitson, 1994) operate within this context. It is reasonable to assume that these socialized expectations about what it means to be a man might contribute to 
adolescent football players becoming conflicted about appropriate ways to engage in the often violent instrumental aggression that is sanctioned and encouraged within a contact sport like football. Subsequently, although the particular variable of masculinity socialization in our current study (i.e., gender role conflict) did not significantly predict on-field moral functioning (nor did it mediate the relationship with moral atmosphere), other related yet distinctly measured constructs of masculinity socialization (e.g., Conformity to Masculine Norms Inventory, Mahalik et al., 2003; Meanings of Adolescent Masculinity Scale, Oransky \& Fisher, 2009) could be used in efforts to more effectively capture this phenomenon. By doing so, future research can explore whether norms of masculinity socialization within sport influence a young student athlete's moral functioning, both on the field and off the field.

As it relates to our third hypothesis, the results of our study did not demonstrate significant mediation between moral atmosphere and moral functioning. However, to better explain this dynamic, future research should explore how potential mediation between these variables might be explained by other psychosocial variables not included in this study. In addition to exploring alternative measures of masculinity socialization, attitudes concerning the professionalization of the sport may represent another possible mediator. Visek and Watson (2005) found that hockey players' levels of moral functioning decreased as their perceptions of the professionalization of their sport increased. That is, the higher in the competitive ranks a player ascends, the more likely he is to subscribe to norms that endorse antisocial behaviors on the ice. As it relates to football, research could examine differences in levels of competitive play to determine if perceived professionalism of the sport mediates the relationship between moral atmosphere and moral functioning. Perhaps football players at higher competitive levels who aspire to play at the next level (e.g., college football, professional football) might endorse norms of engaging in on-field antisocial behaviors and "perceive this aggressive behavior as the gateway to professional play" (Visek \& Watson, 2005, p. 188). Thus, to enhance our theoretical understanding of this dynamic — and to identify possible areas for intervention-future research should empirically examine other possible mediators of the relationship between moral atmosphere and moral functioning within the unique context of football.

Finally, in spite of our fourth hypothesis to the contrary, athletic identity did not significantly predict on-field moral functioning. However, athletic identity was found to be a significant predictor of masculine gender role conflict. That is, the more a high school football player identified with the athlete role, the greater conflict he felt in attempting to adhere to societal expectations of masculinity. Although not hypothesized, this finding is consistent with past research that suggests an overly salient athletic identity has deleterious consequences off the field (Brewer \& Cornelius, 2001; Brewer et al., 1993; Grove et al., 1997). This finding can contribute to our emerging understanding of male student athletes' masculinity socialization experiences, particularly as it relates to gender role conflict and other constructs within the psychological study of men and masculinity. In addition, these results can assist sport psychologists in creating interventions designed to help student athletes cultivate a balanced sense of identity, particularly adolescent male football players who are attempting to simultaneously internalize societal expectations of what it means to be an athlete and what it means to be a man. 


\section{Limitations}

This study has a number of limitations to note. First, the design is cross-sectional and therefore places limits on firmly establishing causality. Further, the experience of football players at these three particular high schools in the Midwest may not be generalizable to other high school football players in different geographic regions or from different backgrounds. Because the study's sample was almost entirely White $(83 \%)$, this study did not explore differences among football players based on race, ethnicity, or culture. Contextual explorations into men's experiences with gender role conflict would benefit from identifying masculinity differences that may be culturally bound (Wester, 2008). Thus, future research should use the unique context of sport to explore racial, ethnic, and cultural expressions of masculinity. An additional methodological limitation was the use of self-report measures. Although self-report vignettes have been effective in examining social behavior in sport, social desirability could influence these subjective self-reports (Kavussanu et al., 2009). Although we attempted to control for social desirability, this is still a limitation, so future studies could benefit from incorporating behavioral observations into their design. In addition, future studies could also use video clips instead of vignettes to provide players with visual representations of the scenarios of the on-field antisocial behaviors. In sum, results should be interpreted in light of these limitations.

\section{Conclusion}

By extending Kavussanu et al.'s (2002) framework into the domain of football, this study represents the first exclusive examination of moral functioning in football, a contact sport that is characterized by its aggressive and often violent nature (Gage, 2008; Messner, 1990). The results of this study suggest that the on-field moral functioning process of high school football players is closely related to the moral atmosphere they perceive to exist on their team. This moral atmosphere is embodied by player perceptions of how coaches think they should behave, as well as perceptions of how fellow teammates would act in similar situations. These results can inform interventions that aim to educate coaches about the impact they can have on influencing how their players play the game. This understanding can help sport psychologists conduct trainings with coaches and administrators so that they can provide optimal growth experiences to the young men whose lives these coaches so greatly impact (Steinfeldt et al., 2010). Coaches can have a direct effect by influencing individual players, yet they may also have an indirect effect by influencing all teammates so that a culture of ethical play can be cultivated on the team and spread from the top down. The ensuing benefits of this culture of prosocial decision-making not only impacts players' development of moral functioning on the field, but it may also have an impact on players' development of moral decision-making skills in life.

\section{Acknowledgments}

The authors would like to thank Jake Blumberg, Mia Morrison, and Erin Pshenishny for their efforts in entering data for this study. The authors would also like to thank Julie LaFollette for her diligent efforts in editing the final version of the manuscript. 


\section{References}

Blazina, C., Pisecco, S., \& O'Neil, J.M. (2005). An adaptation of the Gender Role Conflict Scale for adolescents: Psychometric issues and correlates with psychological distress. Psychology of Men \& Masculinity, 6(1), 39-45.

Blazina, C., \& Watkins, C.E. (1996). Masculine gender role conflict: Effects on college men's psychological well-being, chemical substance usage, and attitudes toward help-seeking. Journal of Counseling Psychology, 43(4), 461-465.

Bredemeier, B.J., \& Shields, D.L. (1986). Game reasoning and interactional morality. The Journal of Genetic Psychology, 147(2), 257-275.

Brewer, B.W., \& Cornelius, A.E. (2001). Norms and factorial invariance of the Athletic Identity Measurement Scale. Academic Athletic Journal, 15, 103-113.

Brewer, B.W., Van Raalte, J.L., \& Linder, D.E. (1993). Athletic identity: Hercules muscle or Achilles heel? International Journal of Sport Psychology, 24(2), 235-254.

Cohn, A.M., \& Zeichner, A. (2005). Effects of masculine identity and gender role stress on aggression in men. Psychology of Men \& Masculinity, 7(4), 179-190.

Cohn, A.M., Jakupcak, M., Seibert, L.A., Hildebrandt, T.B., \& Zeichner, A. (2010). The role of emotion dysregulation $\mathrm{n}$ the association between men's restrictive emotionality and use of physical aggression. Psychology of Men \& Masculinity, 11(1), 53-64.

Cornelius, A. (1995). The relationship between athletic identity, peer and faculty socialization, and college student development. Journal of College Student Development, 36(6), 560-573.

Coulomb-Cabagno, G., \& Rascle, O. (2006). Team sports players' observed aggression as a function of gender, competitive level, and sport type. Journal of Applied Sport Psychology, 36(8), 1980-2000.

Cournoyer, R.J., \& Mahalik, J.R. (1995). Cross-sectional study of gender role conflict examining college-aged and middle-aged men. Journal of Counseling Psychology, 42(1), 11-19.

Gage, E.A. (2008). Gender attitudes and sexual behaviors: Comparing center and marginal athletes and nonathletes in a collegiate setting. Violence Against Women, 14(8), 1014-1032.

Greendorfer, S.L. (1993). Gender stereotypes and early childhood socialization. In G.L. Cohen (Ed.), Women in sport: Issues and controversies (pp. 3-14). Newbury Park, CA: Sage.

Grove, J.R., Lavallee, D., \& Gordon, S. (1997). Coping with retirement from sport: The influence of athletic identity. Journal of Applied Sport Psychology, 9(2), 191-203.

Guttman, L. (1945). A basis for analyzing test-retest reliability. Psychometrika, 10, 255-282.

Kavussanu, M., Roberts, G.C., \& Ntoumanis, N. (2002). Contextual influences on moral functioning of college basketball players. The Sport Psychologist, 16(4), 347-367.

Kavussanu, M., Stamp, R., Slade, G., \& Ring, C. (2009). Observed prosocial and antisocial behaviors in male and female soccer players. Journal of Applied Sport Psychology, 21(1), S62-S76.

Kreager, D.A. (2007). Unnecessary roughness: School sports, peer networks, and male adolescent violence. American Sociological Review, 72(5), 705-724.

Little, T.D., Cunningham, W.A., Shahar, G., \& Widaman, K.F. (2002). To parcel or not to parcel: Exploring the question, weighing the merits. Structural Equation Modeling, 9(2), 151-173.

MacKinnon, D.P., Lockwood, C.M., \& Williams, J. (2004). Confidence limits for the indirect effect: Distribution of the product and resampling methods. Multivariate Behavioral Research, 39(1), 99-128.

Mahalik, J.R., Locke, B., Ludlow, L., Diemer, M., Scott, R.P.J., Gottfried, M., et al. (2003). Development of the Conformity to Masculine Norms Inventory. Psychology of Men \& Masculinity, 4(1), 3-25. 
McDonald, R.P., \& Ho, M. (2002). Principles and practice in reporting structural equation analyses. Psychological Methods, 7(1), 64-82.

Messner, M.A. (1990). When bodies are weapons: Masculinity and violence in sport. International Review for the Sociology of Sport, 25(3), 203-220.

Messner, M.A. (1992). Power at play: Sports and the problem of masculinity. Boston: Beacon Press.

Messner, M.A. (2002). Taking the field: Women, men and sports. Minneapolis, MN: UM Press.

Miller, B.W., Roberts, G.C., \& Ommundsen, Y. (2005). Effects of perceived motivational climate on moral functioning, team moral atmosphere perceptions, and the legitimacy of intentionally injurious acts among competitive youth football players. Psychology of Sport and Exercise, 6(4), 461-477.

Miller, P.S., \& Kerr, G. (2002). The athletic, academic, and social experiences of intercollegiate student-athletes. Journal of Sport Behavior, 25(4), 346-367.

Monk, D., \& Ricciardelli, L.A. (2003). Three dimensions of the male gender role as correlates of alcohol and cannabis involvement in young Australian men. Psychology of Men \& Masculinity, 4(1), 57-69.

Muthén, L. K., \& Muthén, B. O. (2010). Mplus (Version 6). Los Angeles, CA: Muthén \& Muthén.

O’Neil, J.M. (2008). Summarizing 25 years of research on men's gender role conflict using the Gender Role Conflict Scale: New research paradigms and clinical implications. The Counseling Psychologist, 36(3), 358-445.

O’Neil, J.M. (2010). Gender role conflict research paradigm. Retrieved online from http:// web.uconn.edu/joneil/GenderHome.html

O’Neil, J.M., Helms, B., Gable, R., David, L., \& Wrightsman, L. (1986). Gender Role Conflict Scale (GRCS): College men's fears of femininity. Sex Roles, 14(5-6), 335-350.

Oransky, M., \& Fisher, C. (2009). The development and validation of the meanings of adolescent masculinity scale. Psychology of Men \& Masculinity, 10(1), 57-72.

Pleck, J.H. (1995). The gender role strain paradigm: An update. In R.F. Levant \& W.S. Pollack (Eds.), A new psychology of men (pp. 11-32). New York: Basic Books.

Rest, J.R. (1983). Morality. In P. Mussen (Ed.), Manual of child psychology. J. Flavell \& E. Markman (Eds.), Vol. 3: Cognitive development (4th ed.; pp. 356-629). New York: Wiley.

Rest. J.R. (1984). The major components of morality. In W. Kurtines \& J. Gewirtz (Eds.), Morality, moral behavior, and moral development (pp. 356-429). New York: Wiley.

Rochlen, A.B., \& Mahalik, J.R. (2004). Women's perceptions of male partners' gender role conflict as predictors of psychological well-being and relationship satisfaction. Psychology of Men \& Masculinity, 5(2), 147-157.

Sage, L., Kavussanu, M., \& Duda, J. (2006). Goal orientations and moral identity as predictors of prosocial and antisocial functioning in male association football players. Journal of Sports Sciences, 24(5), 455-466.

Saris, W., Satorra, A., \& van der Veld, W. (2009). Testing structural equation models or detection of misspecification? Structural Equation Modeling, 16(4), 561-582.

Shepard, D.S. (2002). A negative state of mind: Patterns of depressive symptoms among men with high gender role conflict. Psychology of Men \& Masculinity, 3(1), 3-8.

Shields, D.L., \& Bredemeier, B.L. (2007). Advances in sport morality research. In G. Tenenbaum \& R. Eklund (Eds.), Handbook of Sport Psychology (3rd ed., pp. 662-684). Hoboken, NJ: Wiley \& Sons, Inc.

Shields, D.L., Bredemeier, B.J., Gardner, D.E., \& Bostrom, A. (1995). Leadership, cohesion, and team norms regarding cheating and aggression. Sociology of Sport Journal, 12(3), 324-336.

Shields, D.L., LaVoi, N.M., Bredemeier, B.J., \& Power, F.C. (2007). Predictors of poor sportspersonship in youth sports: Personal attitudes and social influences. Journal of Sport \& Exercise Psychology, 29(6), 747-762. 
Sijtsma, K. (2009). On the use, the misuse, and the very limited usefulness of Cronbach's alpha. Psychometrika, 74, 107-120.

Steinfeldt, J.A., Foltz, B.D., Mungro, J., Speight, Q.L., Wong, Y.J., \& Blumberg, J. (2010, November 15).Masculinity socialization in sports: Influence of college football coaches. Psychology of Men \& Masculinity. Advance online publication. doi:10.1037/a0020170

Steinfeldt, J.A., \& Steinfeldt, M.C. (2010). Gender role conflict, athletic identity, and help-seeking attitudes among high school football players. Journal of Applied Sport Psychology, 22(3), 262-273.

Steinfeldt, J.A., Steinfeldt, M.C., England, B., \& Speight, Q. (2009). Gender role conflict and help-seeking stigma among college football players. Psychology of Men \& Masculinity, 10(4), 261-272.

Stephens, D.E. (1998). Aggression. In J.L. Duda (Ed.), Advances in sport and exercise psychology measurement (pp. 277-292). Morgantown, WV: Fitness Information Technology.

Stephens, D.E., \& Kavanagh, B. (2003). Aggression in Canadian youth ice hockey: The role of moral atmosphere. International Sports Journal, 7(3), 109-119.

Strahan, R., \& Gerbasi, K.C. (1972). Short, homogeneous versions of the Marlow-Crowne Social Desirability Scale. Journal of Clinical Psychology, 28(2), 191-193.

Ten Berge, J., \& Sočan, G. (2004). The greatest lower bound to the reliability of a test and the hypothesis of unidimensionality. Psychometrika, 69(4), 613-625.

Thompson, E.H., \& Pleck, J.H. (1986). The structure of male norms. The American Behavioral Scientist, 29(5), 531-543.

Visek, A., \& Watson, J. (2005). Ice hockey players' legitimacy of aggression and professionalization of attitudes. The Sport Psychologist, 19(2), 178-192.

Watts, R.H., \& Borders, L.D. (2005). Boys' perceptions of the male role: Understanding gender role conflict in adolescent males. Journal of Men's Studies, 13(2), 267-280.

Wester, S.R. (2008). Male gender role conflict and multiculturalism: Implications for counseling psychology. The Counseling Psychologist, 36(2), 294-324.

Whitson, D. (1994). The embodiment of gender: Discipline, domination, and empowerment. In S. Birrell \& C. Cole (Eds.), Women, sport and culture (pp. 353-372). Champaign, IL: Human Kinetics.

Wong, Y.J., Pituch, K.A., \& Rochlen, A.B. (2006). Men's restrictive emotionality: An investigation of associations with other emotion-related constructs, anxiety, and underlying dimensions. Psychology of Men \& Masculinity, 7(2), 113-126.

Wong, Y.J., Steinfeldt, J.A., Speight, Q.L., \& Hickman, S.J. (2010). Content analysis of the Psychology of Men and Masculinity (2000 to 2008). Psychology of Men \& Masculinity, 11(3), 170-182.

Manuscript submitted: February 22, 2010

Revision accepted: November 28, 2010 